\title{
Hands Free Automatic Clinical Care Documentation: Opportunities for Motion Sensors and Cameras
}

\author{
Daniel Fabbri $^{1}$ - Jesse M. Ehrenfeld ${ }^{1}$
}

Published online: 16 August 2016

(C) Springer Science+Business Media New York 2016

Electronic care documentation continues to represent a significant time expense in many clinical environments. The fundamental challenge centers around the need to either (i) pause from the provision of care to document clinical activities, or (ii) document care after the encounter. This serial workflow prevents simultaneous care and documentation, which can result in extensive physician frustration, errors in documentation and clinical errors. As a result, medical scribes are increasingly being used in high throughput clinical areas to reduce the documentation burden on clinicians. However, the addition of personnel to create documentation is resource intensive.

Hands free clinical documentation systems can alleviate these documentation problems [1-3]. Systems that rely on voice recognition have shown some promise [4], but suffer from performance deterioration in settings where (1) there is any significant degree of ambient noise or (2) multiple caregivers are present, (3) most acute care environments where voice commands are indistinguishable from conversations. A new approach to hands free automatic clinical care documentation is therefore needed to improve the functioning of our medical systems.

Given the advances in off-the shelf sensors, such as wristworn motion sensors and cameras, it is possible to automatically generate a clinical care record by detecting the signatures associated with key clinical tasks [5-10]. Wrist-worn sensors contain accelerometers and gyroscopes, which can track mo-

Jesse M. Ehrenfeld

jesse.ehrenfeld@Vanderbilt.Edu

1 Vanderbilt University School of Medicine, Light Hall, Nashville, TN 37232, USA tion and identify clinical interventions such as CPR [11, 12]. Cameras can be used to track physician hand position and their relationship with the patient's body to construct an injury heatmap. Additionally, the fusion of motion sensors and cameras allows for even further opportunities for completing the clinical care record.

Realistically, the addition of motion sensors and cameras will not be able to identify every aspect of clinical care in the near term. However, specific environments and interventions may be amenable to these methods, one of which is patient transport. During patient transport, patient movement is restricted and the set of possible interventions is limited. Moreover, receiving physicians can benefit from real-time and accurate documentation.

If successful, hands free documentation can enable clinicians to focus on the clinical tasks at hand (i.e. the patient in front of them), effectively hand-off patients between providers, and allow receiving physicians to better prepare for patient arrivals.

The medical community must leverage emerging technologies to improve clinical care. The automatic identification and documentation of key clinical concepts is an important next step in optimizing clinician resource utilization.

\section{References}

1. Strauss, D.G., Sprague, P.Q., Underhill, K., Maynard, C., Adams, G.L., Kessenich, A., Sketch Jr., M.H., Berger, P.B., Marcozzi, D., Granger, C.B., and Wagner, G.S., Paramedic transtelephonic communication to cardiologist of clinical and electrocardiographic assessment for rapid reperfusion of ST-elevation myocardial infarction. J. Electrocardiol. 40:265-270, 2007.

2. Thompson, S.J., and Schriver, J.A., A survey of prehospital care paramedic/physician communication for Multnomah County (Portland), Oregon. J. Emerg. Med. 1:421-428, 1984. 
3. McGarry, A.B., Mott, J.C., and Kotwal, R.S., A study of prehospital medical documentation by military medical providers during precombat training. J. Spec. Oper. Med. 15:79-84, 2015.

4. Rosenbloom, S.T., Stead, W.W., Denny, J.C., Giuse, D., Lorenzi, N.M., Brown, S.H., and Johnson, K.B., Generating clinical notes for electronic health record systems. Appl. Clin. Inform. 1:232-243, 2010.

5. Nguyen, H.P., Ayachi, F., Lavigne-Pelletier, C., Blamoutier, M., Rahimi, F., Boissy, P., Jog, M., and Duval, C., Auto detection and segmentation of physical activities during a Timed-Up-and-Go (TUG) task in healthy older adults using multiple inertial sensors. J. Neuroeng. Rehabil. 12:36, 2015.

6. Chandler, J.L., Brazendale, K., Beets, M.W., and Mealing, B.A., Classification of physical activity intensities using a wrist-worn accelerometer in 8-12-year-old children. Pediatr. Obes. 11:120 127, 2016.

7. Amireault, S., Godin, G., Lacombe, J., and Sabiston, C.M., Validation of the Godin-Shephard leisure-time physical activity questionnaire classification coding system using accelerometer assessment among breast cancer survivors. J. Cancer Surviv. 9: 532-540, 2015.

8. Hikihara, Y., Tanaka, C., Oshima, Y., Ohkawara, K., IshikawaTakata, K., and Tanaka, S., Prediction models discriminating between nonlocomotive and locomotive activities in children using a triaxial accelerometer with a gravity-removal physical activity classification algorithm. PLoS One. 9, 2014.

9. Zhang, S.Y., Rowlands, A.V., Murray, P., and Hurst, T.L., Physical activity classification using the GENEA wrist-worn accelerometer. Med. Sci. Sports Exerc. 44:742-748, 2012.

10. Godfrey, A., Bourke, A.K., Olaighin, G.M., van de Ven, P., and Nelson, J., Activity classification using a single chest mounted triaxial accelerometer. Med. Eng. Phys. 33:1127-1135, 2011.

11. Ling, Y., Automatic human daily activity segmentation applying smart sensing technology. Int. J. Smart Sens. Intell. Syst. 8:1624 $1640,2015$.

12. Lampe, J.W., Trasolini, N., Diallo, S., and Becker, L.B., Using Accelerometers To Track Chest Wall Motion During CPR. Circulation. 2010. 